\title{
Delayed puberty and abnormal anthropometry and its associations with quality of life in young Fontan survivors: A multicenter cross-sectional study
}

\author{
Shaji C. Menon MD1 [i] | Ragheed Al-Dulaimi MD, $\mathrm{MPH}^{1}$ । \\ Brian W. McCrindle MD, MPH ${ }^{2}$ | David J. Goldberg MD ${ }^{3} \quad$ Ritu Sachdeva MD ${ }^{4}$ | \\ Bryan H. Goldstein MD5 (1) | Thomas Seery MD ${ }^{6}$ | Karen C. Uzark PhD7 | \\ Anjali Chelliah MD8 | Ryan Butts MD9 | Heather Henderson MD10 | \\ Tiffanie Johnson MD11 | Richard V. Williams MD ${ }^{1}$
}

${ }^{1}$ University of Utah and Primary Children's Hospital, Salt Lake City, Utah, USA

${ }^{2}$ The Hospital for Sick Children, Toronto, Ontario, Canada

${ }^{3}$ Children's Hospital of Philadelphia, Philadelphia, Pennsylvania, USA

${ }^{4}$ Emory University School of Med, Atlanta, Georgia, USA

${ }^{5}$ Cincinnati Children's Hospital Medical Center, Cincinnati, Ohio, USA

${ }^{6}$ Texas Children's Hospital, Houston, Texas, USA

${ }^{7}$ University of Michigan, Ann Arbor, Michigan, USA

${ }^{8}$ Columbia University Medical Center, New York, New York, USA

${ }^{9}$ Medical University of South Carolina, Charleston, South Carolina, USA

${ }^{10}$ Duke University School of Medicine, Durham, North Carolina, USA

${ }^{11}$ Riley Hospital for Children, Indianapolis, Indiana, USA

\section{Correspondence}

Shaji C. Menon, MD, Primary Children's Medical Hospital, 81 N Mario Capecchi Drive, Salt Lake City, UT 84113, USA.

Email: shaji.menon@hsc.utah.edu

\section{Funding information}

Pediatric Heart Network Scholar Funding for Mentored Research

\begin{abstract}
Introduction: We sought to evaluate the prevalence of delayed puberty and abnormal anthropometry and its association with quality of life (QoL) in young Fontan survivors.

Methods: This was a cross-sectional study at 11 Pediatric Heart Network centers. Demographic and clinical data, anthropomety, and Tanner stage were collected. Anthropometric measurements and pubertal stage were compared to US norms. QoL was assessed using Pediatric Quality of Life inventory (PedsQL). Mixed effects regression modeling adjusting for clustering by center was used to evaluate factors associated with abnormal anthropometry and delayed puberty and associations with QoL.

Results: Of the 299 subjects, $42 \%$ were female. The median enrollment age was 13.9 years, and the median age at Fontan was 3 years. Fontan survivors had a higher prevalence of short stature relative to normative data $(20 \%$ vs $5 \%, P<.0001)$ and an increased prevalence of abnormal BMI (16\% vs $10 \%, P<.0001$ ) (low [43\%] and high [57\%]). Fontan subjects, both males (58\%) and females (58\%), had a delay of $1.5-2$ years in $\geq 1$ Tanner stage parameter compared to normal population. There was no association between delayed puberty and QoL. Abnormal anthropometry was associated with lower overall $(62.3 \pm 17.3$ vs $72.5 \pm 16.6 ; P<.001)$ and physical appearance scores $(72.2 \pm 27.4$ vs $79.8 \pm 21.5 ; P<.01)$. Lower exercise capacity was associated with abnormal anthropometry and $>2$ surgeries before Fontan was associated with delayed puberty. Lower family income ( $<\$ 25000)$ and hypoplastic left heart syndrome were associated with lower QoL.

Conclusion: Compared to the normal population, Fontan survivors have high prevalence of short stature, abnormal BMI and delayed puberty. Abnormal anthropometry, but not delayed puberty, was associated with lower overall QoL and perceived physical appearance scores. Routine screening for abnormal anthropometry, especially in HLHS and in lower socioeconomic status families, should be considered to allow interventions, which might ameliorate the negative psychosocial impact.
\end{abstract}

KEYWORDS

abnormal growth, delayed puberty, Fontan, quality of life

Abbreviations: BMI, body mass index; BNP, B-type natriuretic peptide; HLHS, hypoplastic left heart syndrome; NHANES, National Health and Nutrition Examination Survey; NYHA, New York Heart Association; PedsQL, Pediatric Quality of Life inventory; PHN, Pediatric Heart Network; QoL, quality of life. 


\section{1 | BACKGROUND}

Growth failure in infants and children with congenital heart disease, particularly in infants with single ventricle physiology, is a well-known phenomenon. ${ }^{1-3}$ Patients with single ventricle physiology are a heterogeneous group who typically undergo a series of high-risk palliative cardiac surgeries culminating in the Fontan operation. Fontan physiology is inherently associated with a lifelong risk of multiple medical complications including ventricular dysfunction, arrhythmias, stroke, exercise intolerance, protein-losing enteropathy, plastic bronchitis, liver dysfunction, and premature death. ${ }^{4-6}$ These complications may predispose them at high risk for growth and pubertal aberrations. Abnormalities in height and weight have been reported in Fontan survivors ${ }^{7}$ and abnormal anthropometry has been associated with lower functional health status scores. ${ }^{7}$ Previous studies demonstrated long-term growth and cognitive aberrations secondary to growth failure in early life including higher prevalence of short stature, poor school performance, and higher incidence of behavioral and attention issues. ${ }^{8}$ The same factors that predispose single ventricle patients to abnormal anthropometry have also been implicated in the delayed puberty. However, despite the high prevalence of these risk factors, the actual prevalence of delayed puberty in Fontan survivors is unknown. Furthermore, the effects of delayed puberty on quality of life (QoL) have not been studied in Fontan survivors.

We sought to determine the prevalence of abnormal anthropometry and delayed puberty in adolescent Fontan survivors and to define demographic and clinical factors associated with these abnormalities. We also explored the association between abnormal anthropometry and delayed puberty and QoL. We hypothesized that the prevalence of abnormal anthropometry and delayed puberty would be significantly higher in the study population compared to the normal population and these abnormalities would be associated with lower QoL scores.

\section{2 | METHODS}

This study was supported by a grant from the National Heart, Lung, and Blood Institute funded Pediatric Heart Network (PHN). The authors are solely responsible for the design and conduct of this study. All study analyses and the drafting and editing of the article and its final contents were approved by all the authors.

\subsection{Study population}

This was a cross-sectional multicenter study of adolescent Fontan survivors between the ages of 8 and 18 years. All Fontan patients between the ages of 8 and 18 years who consented or whose parent/ guardian consented to participate in the study from $11 \mathrm{PHN}$ centers (02/2013-003/2015) were eligible to participate in the study. Patients or their parent/guardian who could not understand the QoL English forms were excluded. Patients with genetic syndrome were specifically excluded from the study. The study was approved by the Institutional Review Boards at all participating centers.

\subsection{1 | Tanner staging}

Participants Tanner stage was determined by using a self-assessment pictorial Tanner stage questionnaire. Study subjects were given a previously validated standardized series of realistic color images with explanatory text to assess their own pubertal development. ${ }^{9,10}$ Compared with Tanner's original black and white pictures, selfassessment of the pubertal stage by using realistic color images is strongly associated with a clinician's objective assessment of Tanner stage. ${ }^{9}$ Self-reported age of menarche was also recorded.

\section{2 | Definitions}

\subsection{1 | Abnormal anthropometry}

Abnormal anthropometry was defined as presence of either short stature or abnormal BMI. Short stature was defined as height $<5$ th percentile based on the CDC growth curves. ${ }^{11}$ Abnormal BMI was defined as BMI $<5$ th percentile or $\geq 95$ th percentile based on the NHANES III data.

\subsection{2 | Delayed puberty}

Delayed puberty was clinically defined as failure to reach a Tanner stage at an age that is 2 standard deviation greater than the population mean for that specific Tanner stage obtained from the NHANES III data. ${ }^{12,13}$

\subsubsection{Q Quality of life (QoL)}

QoL was assessed using a previously validated Pediatric Quality of Life Inventory (PedsQL) and Cardiac Module. We chose to use PedsQL for this study because it is shorter, easier to complete compared to other instruments and provides both the child's and the parent's perspectives across the entire study age range. ${ }^{14}$ PedsQL was also a preferred instrument for QoL testing because its generic core scale has been obtained from a large normative database of ethnically diverse healthy children as well as children with chronic health conditions. ${ }^{14}$ The $23-$ item PedsQL generic core scales encompass physical functioning (eight items), emotional functioning (five items), social functioning (five items), and school functioning (five items). The PedsQL 3.0 cardiac module has five scales related to symptoms, perceived physical appearance, treatment anxiety, cognitive problems, and communication for parent proxy report and children aged $8-18$ years.

\subsubsection{Socioeconomic status}

Socioeconomic status was assessed using a parent-completed form that collected information regarding family income, race and ethnicity, education of the primary caregiver, family marital status, and employment of the primary caregiver.

\subsection{5 | Normative data}

Normative data for height were obtained from CDC growth curves. Normative data for BMI and Tanner stages were obtained from National Health and Nutrition Examination Survey (NHANES III). The NHANES surveys were designed to give national normative estimates of the health and nutritional status of the US civilian, noninstitutionalized population. NHANES III was conducted from 1988 to 1994 using a stratified, multistage probability design. 
The medical record was reviewed for demographics, cardiac anatomic diagnosis, type of surgical palliation, details of Fontan, and other surgical palliation including the timing, presence of ventricular dysfunction and atrioventricular valve regurgitation, medications, exercise stress test results, New York Heart Association (NYHA) Functional Classification, and B-natriuretic peptide measurements (BNP). All data included in the study were collected within 6 months of study participation.

\section{3 | Statistical analysis}

Continuous variables are described as mean \pm standard deviation or median (interquartile range). BMI and short stature were treated as categorical variables, based on the definition above. To evaluate the differences between subjects with abnormal measures (BMI and height) from the normal population, one-sample Z-test was performed. For each of the Tanner stages, inverse variance weighting method to pool the mean age difference across strata was used and then Z-test was used to examine the statistical significance for the overall effect. Comparisons between self-reported and parent reports (proxy report) mean generic and domain scores and cardiac module domain score were performed using a paired $T$-test and Lin Index for concordance correlation coefficient. A mixed effects regression model adjusting for clustering by center was performed to evaluate associations between demographic, anatomic, and clinical factors and abnormal anthropometry and delayed puberty. A backward elimination method was used for variable selection before performing the logistic regression. Similarly, to study the associations between delayed puberty and abnormal anthropometry and QoL, a mixed effects regression model adjusting for clustering by center was performed. In studies with multiple centers, we sometimes expect patient outcomes to differ according to center. This could be due to differences between patients who present to different centers, or because of differences between the centers themselves. To minimize the impact of any between-center differences on the study results, adjustment for clustering is performed to account for centereffects in the analysis model. This mixed effect model included basic demographic variables, family income, race, ethnicity, education of the primary caregiver, family marital status, and employment of the primary caregiver. All analyses were conducted using SAS version 9.4 (SAS Institute, Inc, Cary, North Carolina).

\section{3 | RESULTS}

Of the 299 subjects enrolled in the study, 33\% had hypoplastic left heart syndrome (HLHS). The median age of the study cohort was 13.9 years (IQR: 11.3, 16.1 years). Baseline characteristics of the study population are shown in Table 1.

\section{1 | Abnormal anthropometry}

\subsection{1 $\mid \mathrm{BMI}$}

Compared to a $10 \%$ prevalence of abnormal BMI in the normal US population, $16 \%$ of adolescent Fontan survivors had abnormal BMI
TABLE 1 Baseline characteristics of study cohort $(n=299)$

\begin{tabular}{|c|c|}
\hline Females & $125(42 \%)$ \\
\hline \multicolumn{2}{|l|}{ Age groups (years) } \\
\hline $8-12$ & $41 \%$ \\
\hline $13-15$ & $33 \%$ \\
\hline$\geq 16$ & $26 \%$ \\
\hline \multicolumn{2}{|l|}{ Race } \\
\hline White & $224(75 \%)$ \\
\hline Black & $35(12 \%)$ \\
\hline Others & $40(13 \%)$ \\
\hline \multicolumn{2}{|l|}{ Ethinicity } \\
\hline Hispanic & $43(14 \%)$ \\
\hline Non-Hispanic & $255(86 \%)$ \\
\hline Hypoplastic left heart syndrome, N (\%) & $98(33 \%)$ \\
\hline Heterotaxy, N (\%) & $24(8 \%)$ \\
\hline \multicolumn{2}{|l|}{ Ventricular morphology } \\
\hline Left ventricle dominant & 152 (50\%) \\
\hline Right ventricle dominant & $136(46 \%)$ \\
\hline Mixed ventricular morphology & $11(4 \%)$ \\
\hline Median age at enrollment (years) (IQR) & $13.9(11.3,16.1)$ \\
\hline Median age at Fontan (years) (IQR) & $3(2,4)$ \\
\hline Median age at Glenn (months) (IQR) & $4(3,5)$ \\
\hline Median weight at Fontan in kg (IQR) & $13.7(11.9,15.3)$ \\
\hline Mean height at Fontan in $\mathrm{cm}$ (SD) & $93.3( \pm 15.2)$ \\
\hline Median number of surgeries before & $2(1-3)$ \\
\hline $\begin{array}{l}\text { Fontan (range) } \\
>2 \text { Cardiac surgeries }\end{array}$ & $96(32 \%)$ \\
\hline \multicolumn{2}{|l|}{ Type of Fontan } \\
\hline Extra/intracardiac conduit & 199 (67\%) \\
\hline Lateral tunnel & $85(29 \%)$ \\
\hline Hemi-Fontan & $56(19 \%)$ \\
\hline Kawashima & $6(2 \%)$ \\
\hline Other Fontan types & $9(4 \%)$ \\
\hline \multicolumn{2}{|l|}{ Post-Fontan complications } \\
\hline Pacemaker & $45(15 \%)$ \\
\hline Stroke & $33(11 \%)$ \\
\hline PLE & $15(5 \%)$ \\
\hline Cirrhosis & $3(1 \%)$ \\
\hline Arrhythmia & $39(13 \%)$ \\
\hline Thrombosis & $20(7 \%)$ \\
\hline Seizure & $10(3 \%)$ \\
\hline \multicolumn{2}{|l|}{ Severity of ventricular dysfunction $(N=270)$} \\
\hline Mild & $17(6 \%)$ \\
\hline Moderate & $5(2 \%)$ \\
\hline Severe & $7(2 \%)$ \\
\hline \multicolumn{2}{|l|}{ Severity of AV valve regurgitation $(N=268)$} \\
\hline Mild & 118 (39\%) \\
\hline Moderate & $34(11 \%)$ \\
\hline Severe & $3(1 \%)$ \\
\hline NYHA grades III and IV & $30(10 \%)$ \\
\hline$\%$ predicted peak $\mathrm{VO}_{2} \max (N=102)$ & $67.5 \pm 15.4$ \\
\hline $\mathrm{BNP} \mathrm{pg} / \mathrm{ml}(\mathrm{N}=39)$ & $29.1(15.9,139.2)$ \\
\hline Pulmonary vasodilators & $15(5 \%)$ \\
\hline Coumadin & 32 (11\%) \\
\hline Digoxin & $30(10 \%)$ \\
\hline ACE inhibitors & $156(52 \%)$ \\
\hline Beta-blockers & $34(11 \%)$ \\
\hline Diuretics & $48(16 \%)$ \\
\hline Mean age at menarche in years $(\mathrm{SD})(N=76)$ & $12.5( \pm 1.7)$ \\
\hline Mean height Z-score & $-0.7( \pm 1.6)$ \\
\hline BMI Z-score & $-0.01( \pm 1.9)$ \\
\hline
\end{tabular}

For missing values, we have added number of subjects in column 1. Abbreviations: $\mathrm{ACE}$, angiotensin-converting enzyme; $\mathrm{AV}$, atrioventricular; BMI, body mass index; BNP, B-natiuretic peptide; NYHA: New York Heart Association; PLE, protein-losing enteropathy. 
Mean difference in age in years at different Tanner stages between study population and NHANES III

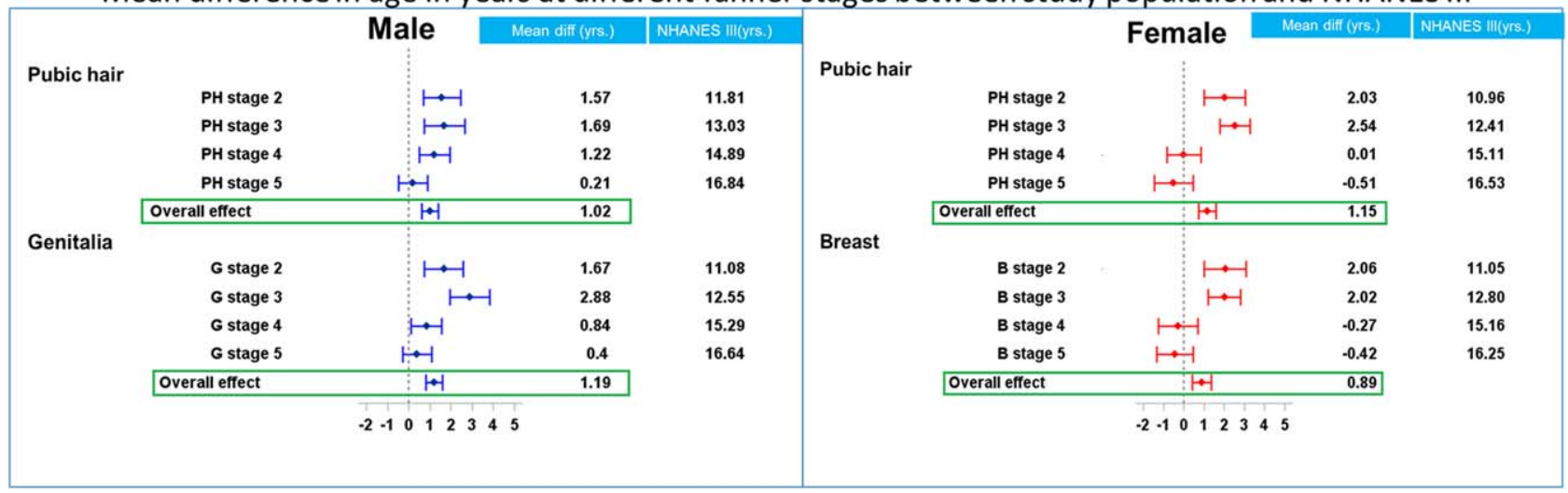

FIGURE 1 Shown in this forest plot are the mean differences in years between the Fontan study cohort and the normal population (NHANES III) in achieving various Tanner stages. The mean age at which the normal population achieves a specific Tanner stage is shown in the last column

$(P<$.0001). Of the Fontan survivors who had an abnormal BMI, 43\% had $\mathrm{BMI}<5$ th percentile and $57 \%$ had $\mathrm{BMI}>95$ th percentile.

\subsection{2 | Short stature}

The prevalence of short stature in adolescent Fontan survivors was higher relative to the normal population. In the study cohort, $20 \%$ of participants had short stature compared to $5 \%$ in the normal population $(P<.0001)$

\subsection{3 | Delayed puberty}

More than half of the study population (males: $58 \%$, females: $58 \%$ ) had delay in at least one Tanner stage parameter. Pubic hair growth was delayed in $43 \%$ of males and $47 \%$ of females. Genitalia development was delayed in $54 \%$ of males, and breast development was delayed in $47 \%$ of females. A delay in both pubic hair growth and genitalia/breasts development was seen in $39 \%$ of males and $36 \%$ of females. On average, both in male and female Fontan survivors, there was a delay of 1.5-2 years in the achievement of Tanner stages compared to the normal population (Figure 1).

\subsection{4 | QoL}

There was a moderate agreement between self-reported and proxy (parent reported) QoL scores (Figure 2). However, parent QoL scores were lower than the child/adolescent's scores (68.9 vs $72.6 ; P<.01)$ for the majority of domains in generic core scale and cardiac module. In Fontan survivors, abnormal anthropometry was associated with lower QoL (generic core scale total score: $62.3 \pm 17$ vs $72.5 \pm 16.6$; $P<.001$ ) (Figure $2 A$ and $B$ ) and lower cardiac module perceived physical appearance scores on proxy report (72.2 \pm 27.4 vs $79.8 \pm 21.5$; $P<$.01) (Figure 2D). Delayed puberty was not associated with lower QoL (generic core scale total score: $68.3 \pm 17.4$ vs $70 \pm 17.5 ; P=.4$ ) (Figure 2C).

\subsection{5 | Factors associated with abnormal anthropometry and delayed puberty}

NYHA Class III and IV and lower oxygen consumption on exercise stress test were independently associated with higher risk of abnormal anthropometry (Table 2). More than two cardiac surgeries before Fontan procedure were the only factor associated with higher risk of delayed puberty. Importantly, ventricle morphology, weight-for-age zscore, age at any surgical palliation procedure, cardiac diagnoses, atrioventricular valve regurgitation, use of digoxin therapy, use of pulmonary vasodilator therapy, BNP level, and number of Fontan complications were not associated with either abnormal anthropometry or delayed puberty.

\subsubsection{Association between abnormal anthropometry and delayed puberty and QoL}

On multivariable mixed effects modeling controlling for clustering among centers, delayed puberty was not associated with lower QoL. Factors independently associated with lower overall QoL score were abnormal anthropometry, family income $<\$ 25000$, and HLHS. Female sex and lower family income were independently associated with lower perceived physical appearance scores (Table 2).

\section{4 | DISCUSSION}

This is the first study to evaluate the prevalence of delayed puberty and its impact on QoL in young Fontan survivors. More than half of the Fontan survivors had delay in one of the Tanner stage parameters, and in both sexes, there was a delay of 1.5-2 years between Fontan survivors and the normal population in achievement of earlier Tanner stages. Even though pubertal attainment was noted to be slower in children with Fontan than in healthy controls, it was not associated with lower overall QoL. Instead, abnormal anthropometry was associated with lower overall QoL and perceived physical appearance scores.

\section{1 | Delayed puberty}

Many chronic diseases are known to be associated with delayed puberty. ${ }^{15}$ In chronic diseases, delayed puberty is usually accompanied by a delay in physical growth and the pubertal growth spurt. ${ }^{15}$ The etiology of delayed puberty in Fontan survivors is likely to be 


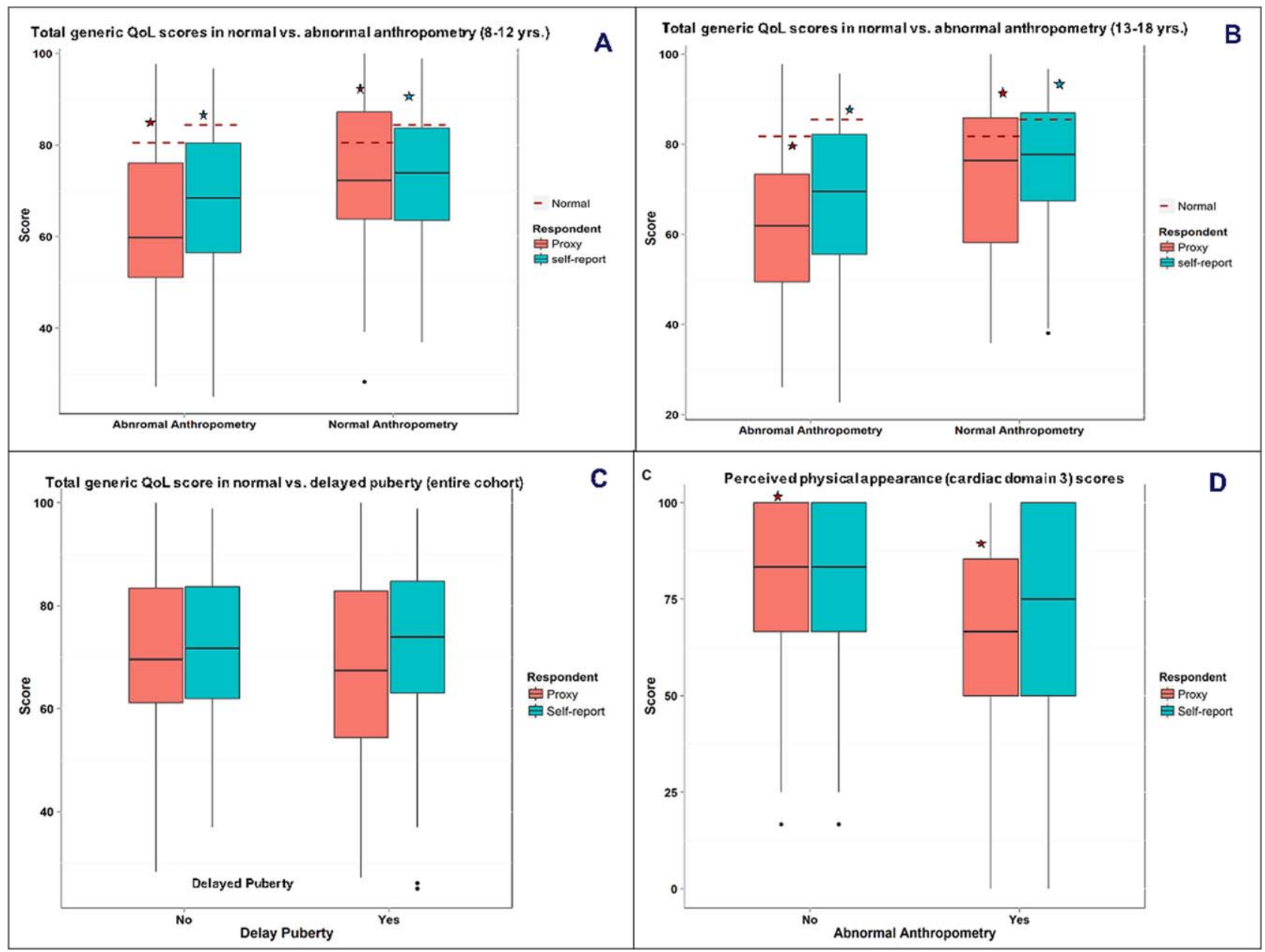

FIGURE 2 (A, B) Box and whisker plots depict the total generic QoL scores for subjects with normal versus abnormal anthropometry stratified by two age groups. The dotted red line indicates the mean score for normal children in the same age group. (C) Shows the total generic QoL scores for normal subjects versus those with delayed puberty for the entire study cohort. (D) Depicts the difference in perceived physical appearance score (cardiac module) for subjects with normal versus abnormal anthropometry.

* represent statistically significant difference with $P$ value $<.05$

multifactorial and may include chronic low cardiac output state and cyanosis, multiple cardiopulmonary bypass, endocrine abnormalities, and Fontan-associated complications, such as Fontan associated liver disease and protein-losing enteropthy. ${ }^{4-6}$ More than two cardiac surgeries before Fontan palliation were the only factor independently associated with delayed puberty in this study. We speculate that multiple cardiopulmonary bypass surgeries early in childhood may result in hypothalamic-pituitary gonadal axis malfunction. Although cardiopulmonary bypass associated hypothalamicpituitary-adrenal axis malfunction is a known phenomenon, the effects of cardiac bypass surgery on gonadal function have not been evaluated. ${ }^{16}$ Future studies should evaluate the effects of multiple cardiopulmonary bypass surgeries on adolescent gonadal function and integrity of hypothalamic-pituitary-adrenal axis.

In this study, we did not find an association between delayed puberty and QoL scores. A possible explanation for this finding is that Fontan survivors have significantly lower QoL compared to peers secondary to a multitude of health issues and delayed puberty may not be perceived by them as a significant concern. An alternate explanation for the lack of association between delayed puberty and QoL may be secondary to the fact that even though the onset of puberty was delayed, older participants achieved complete pubertal development (Tanner stage 5). Since delayed puberty was not associated with worse QoL scores in Fontan survivors, the rational for the evaluation and management of delayed puberty and hypogonadism in this population may be improving fertility and bone mineralization and achievement of height potential. Although, at present, majority of women with Fontan are advised against pregnancy, with improvement in their management could change this recommendation in the future.

\section{2 | Abnormal anthropometry}

\subsection{1 | Short stature}

Compared to the normal population, Fontan survivors have a fourfold increase in the prevalence of short stature and nearly twofold higher abnormality in their BMI. These findings are similar to previous reports of abnormal BMI and short stature in Fontan survivors. ${ }^{7,17,18}$ Short stature was seen in $20 \%$ of young Fontan survivors in this study, which 
TABLE 2 Results of multivariable mixed effect regression analysis accounting for clustering showing factors associated with abnormal anthropometry, delayed puberty, and QoL scores

\section{Adjusted OR}

$95 \% \mathrm{Cl}$

$P$ value

Factors associated with abnormal anthropometry $(\mathrm{AIC}=115.3)$

NYHA grades III and IV vs lower 10.9

Oxygen consumption

10.9

1.8

124.3

0.046

Factors associated with delayed puberty (AIC $=230.5$ )

Cardiac surgeries before Fontan $>2$ vs $\leq 2$

2.98

1.45

6.5

0.02

Factors associated with PedsQL scores

Estimate

$95 \% \mathrm{Cl}$

$P$ value

Self-report (total score) $\left(R^{2}=0.132\right)$

Abnormal anthropometry

Family income $<\$ 25000$ (ref: $\geq \$ 75000$ )

HLHS vs others

$\begin{array}{rr}-7.4 & -11.3 \\ -9.1 & -16 . \\ -4.5 & -8.3\end{array}$

-11.3
-16.4
-8.3

$-3.6$

$-1.7$

$-0.6$

$<.001$

$-4.5$

$-8.3$

.01

.02

Proxy report (total score) $\left(R^{2}=0.157\right)$

Abnormal anthropometry

$-9.2$

$-13.5$

$-18.3$

$-4.9$

$-1.8$

$<.001$

Family income $<\$ 25000$

$-10.0$

$-8.5$

0.03

.01

.05

Self-report (cardiac domain 3: physical appearance) $\left(R^{2}=0.079\right)$

Male vs female

Abnormal anthropometry

Family income $\$ 25000-\$ 49999$

Family income $<\$ 25000$

8.2
-5.3
-11.2
-12.4

2.2

$-11.5$

$-21.5$

$-24.4$

Proxy report (cardiac domain 3: physical appearance) $\left(R^{2}=0.138\right.$ )

Abnormal anthropometry

Family income $\$ 50$ 000-\$74 999

Family income $\$ 25000-\$ 74999$

Family income $<\$ 25000$

$-12.6$

$-16.5$

$-14.2$

$-19.4$

$-18.8$

$-26.5$

$-24.6$

$-31.6$
14.2

0.98

$-0.98$

$-0.3$

.007

.1

.03

.04 is similar to previous report of a quarter of young Fontan survivors with short stature. ${ }^{7}$ Similar to the possible etiologies for delayed puberty, short stature in Fontan patients may be secondary to the multisystem abnormalities. In other chronic diseases, a slowing of growth velocity and a delayed pubertal growth spurt observed in early adolescence may lead to short adult height. ${ }^{15,19}$

\subsubsection{Abnormal BMI}

Similar to findings of this study, increased prevalence of abnormal BMI in Fontan patients has been reported previously. ${ }^{17,18}$ The abnormal BMI was almost equally split between low BMI (43\%) and high BMI (57\%). Not surprisingly, reduced exercise performance (NYHA grades III and IV and lower maximal oxygen uptake) was associated with abnormal anthropometry. This finding suggests a possible role of reduced cardiac output state as an etiology for anthropometric abnormalities in Fontan patients. It is widely believed that short children can suffer from physical, social, and psychological problems. ${ }^{20,21}$ Our findings are similar to those reported by previous studies in that the Fontan survivors with shorter stature and high BMI have poorer functional heath status. ${ }^{17}$ We also noted that abnormal anthropometry was associated with lower self-reported QoL. Association of abnormal anthropometry with lower parent reported physical appearance domain score (cardiac module) but not with self-reported score is an interesting and new finding and further points to the differences in health perceptions between children and their parents.
It is not surprising that Fontan survivors in this study have significantly lower QoL compared to their peers. ${ }^{5,13,22-24}$ Multiple prior reports have shown reduced QoL in patients with significant congenital heart diseases including patients with single ventricle physiology and Fontan. ${ }^{23}$ Our study finding of worse parent's perceptions of QoL status of their children compared to the children's perceptions after the Fontan procedure has been reported before. ${ }^{24}$ Lower family income was associated with lower health-related QoL in Fontan survivors. In fact, a family income of $<\$ 25000$ was associated with a greater reduction in QoL compared to abnormal anthropometry. Other report have shown positive correlations between income and health-related quality of life (QoL). ${ }^{25}$ In this study, HLHS was associated with lower QoL compared to non-HLHS patients and this could be related to the higher mortality and morbidity associated with HLHS. This finding is in contrast to a previous study that reported no difference in QoL between HLHS and non-HLHS Fontan survivors. ${ }^{22}$

\section{3 | Limitations}

There is potentially a selection bias with a healthier population of Fontan survivors more likely consenting to study participation. However, the prevalence of Fontan-associated complications in this study cohort is similar to contemporary reports, indicating that the study population is a representative sample of the contemporary Fontan cohort. The consent rate at most centers was more than $90 \%$ minimizing the selection bias. The study population was primarily Caucasian, and 
generalizability of pubertal findings that are influenced by race/ethnicities to other groups is unknown.

\section{5 | CONCLUSION}

Compared to the normal population, Fontan survivors have a fourfold higher prevalence of short stature and nearly twofold higher prevalence of abnormal BMI. Delayed puberty is common among Fontan survivors of both genders. More than half of the Fontan survivors (58\%) had delay in one of the Tanner stage parameters and on an average both in males and females, there was a delay of $1.5-2$ years in the achievement of earlier Tanner stages compared to the normal population. Abnormal anthropometry, but not delayed puberty, was associated with lower overall QoL and perceived physical appearance scores. Routine screening for abnormal anthropometry, especially in HLHS and in lower socioeconomic status families, should be considered to allow interventions that might ameliorate the negative psychosocial impact.

\section{CONFLICT OF INTEREST}

None.

\section{AUTHOR CONTRIBUTIONS}

Research design, acquisition, analysis or interpretation of data; drafting the article; revising it critically; and approval of the submitted and final versions: Menon, Al-Dulaimi, McCrindle, Goldberg, Sachdeva, Goldstein, Seery, Uzark, Chelliah, Butts, Henderson, Johnson, Williams

\section{ORCID}

Shaji C. Menon MD (iD http://orcid.org/0000-0002-0421-0203

Bryan H. Goldstein MD (DD http://orcid.org/0000-0001-8508-9523

\section{REFERENCES}

[1] Davis D, Davis S, Cotman K, et al. Feeding difficulties and growth delay in children with hypoplastic left heart syndrome versus d-transposition of the great arteries. Pediatr Cardiol. 2008;29(2): 328-333.

[2] Forchielli ML, McColl R, Walker WA, Lo C. Children with congenital heart disease: a nutrition challenge. Nutr Rev. 1994;52(10):348-353.

[3] Anderson JB, Beekman RH, 3rd, Eghtesady P, et al. Predictors of poor weight gain in infants with a single ventricle. J Pediatr. 2010; 157(3):407-413, 413.e1.

[4] Gentles TL, Gauvreau K, Mayer JE, Jr, et al. Functional outcome after the Fontan operation: factors influencing late morbidity. J Thorac Cardiovasc Surg. 1997;114(3):392-403, discussion 404-5.

[5] van den Bosch AE, Roos-Hesselink JW, Van Domburg R, Bogers AJ, Simoons ML, Meijboom FJ. Long-term outcome and quality of life in adult patients after the Fontan operation. Am J Cardiol. 2004;93 (9):1141-1145.

[6] Khairy P, Fernandes SM, Mayer JE, Jr, et al. Long-term survival, modes of death, and predictors of mortality in patients with Fontan surgery. Circulation. 2008;117(1):85-92.

[7] Cohen MI, Bush DM, Ferry RJ, Jr, et al. Somatic growth failure after the Fontan operation. Cardiol Young. 2000;10(05):447-457.
[8] Dykman RA, Casey PH, Ackerman PT, McPherson WB. Behavioral and cognitive status in school-aged children with a history of failure to thrive during early childhood. Clin Pediatr. 2001;40(2):63-70.

[9] Sun Y, Tao FB, Su PY, China Puberty Research C. Self-assessment of pubertal Tanner stage by realistic colour images in representative Chinese obese and non-obese children and adolescents. Acta Paediatr. 2012;101(4):163-166.

[10] Carel JC, Leger J. Clinical practice. Precocious puberty. N Engl J Med. 2008;358(22):2366-2377.

[11] CDC growth charts. Available at http://www.cdc.gov/growthcharts/ cdc_charts.htm. 2009. Accessed February 20, 2018.

[12] Sun SS, Schubert CM, Chumlea WC, et al. National estimates of the timing of sexual maturation and racial differences among US children. Pediatrics. 2002;110(5):911-919.

[13] Palmert MR, Dunkel L. Delayed puberty. N Engl J Med. 2012;366(5): 443-453.

[14] Uzark K, Zak V, Shrader P, et al. Assessment of quality of life in young patients with single ventricle after the Fontan operation. J Pediatr. 2015;170:166-172.e1.

[15] Pozo J, Argente J. Delayed puberty in chronic illness. Best Pract Res Clin Endocrinol Metab. 2002;16(1):73-90.

[16] Bremner WF, Taylor KM, Baird S, et al. Hypothalamo-pituitary-thyroid axis function during cardiopulmonary bypass. J Thorac Cardiovasc Surg. 1978;75(3):392-399.

[17] McCrindle BW, Williams RV, Mital S, et al. Physical activity levels in children and adolescents are reduced after the Fontan procedure, independent of exercise capacity, and are associated with lower perceived general health. Arch Dis Child. 2007;92(6):509-514.

[18] Pinto NM, Marino BS, Wernovsky G, et al. Obesity is a common comorbidity in children with congenital and acquired heart disease. Pediatrics. 2007;120(5):e1157-e1164.

[19] Toumba M, Sergis A, Kanaris C, Skordis N. Endocrine complications in patients with Thalassaemia Major. Pediatr Endocrinol Rev. 2007;5: 642-648

[20] Voss LD, Wiklund I. Short stature and psychosocial assessment. Acta Paediatr Suppl. 1995;411:69-74

[21] Bannink EM, Raat H, Mulder PG, de Muinck Keizer-Schrama SM. Quality of life after growth hormone therapy and induced puberty in women with Turner syndrome. J Pediatr. 2006;148(1):95-101.

[22] Idorn L, Jensen AS, Juul K, et al. Quality of life and cognitive function in Fontan patients, a population-based study. Int J Cardiol. 2013;168(4):3230-3235.

[23] Knowles RL, Day T, Wade A, Bull C, Wren C, Dezateux C. Patientreported quality of life outcomes for children with serious congenital heart defects. Arch Dis Child. 2014;99(5):413-419.

[24] Lambert LM, Minich LL, Newburger JW, et al. Parent- versus child-reported functional health status after the Fontan procedure. Pediatrics. 2009;124(5):e942-e949.

[25] Short P, Mallonee EL. Income disparities in the quality of life of cancer survivors. Med Care. 2006;44(1):16-23.

How to cite this article: Menon SC, Al-Dulaimi R, McCrindle BW, et al. Delayed puberty and abnormal anthropometry and its associations with quality of life in young Fontan survivors: A multicenter cross-sectional study. Congenital Heart Disease. 2018;13:463-469. https://doi.org/10.1111/chd.12597 